\title{
Shared Literacies: Promoting Thinking and Learning in Art Education Through Movement Literacy
}

\author{
Shlomit Ofer \\ Department of Dance, Kibbutzim College of Education Technology and Arts, Tel Aviv, Israel \\ Email address: \\ shlomit.ofer@smkb.ac.il \\ To cite this article: \\ Shlomit Ofer. Shared Literacies: Promoting Thinking and Learning in Art Education Through Movement Literacy. International Journal of \\ Literature and Arts. Vol. 7, No. 4, 2019, pp. 78-86. doi: 10.11648/j.ijla.20190704.12
}

Received: April 6, 2019; Accepted: August 22, 2019; Published: August 27, 2019

\begin{abstract}
The notion of Shared Literacies describes a process in which specialization in two separate domains merge to generate an opportunity for a new kind of knowledge to evolve. The paper presents a study where "movement literacy" (expertise in the field of dance in both theoretical and practical aspects) meets "graphic-symbolic literacy" (expertise in the creation and decoding of graphic-symbolic representations of any kind of knowledge). The theoretical background contains an overview of the two kinds of literacy, as well as two pertinent pedagogical ideas: the ability to translate information between different modes of representation; and self-generation of representations, an idea which emphasizes the independent design of symbols by learners. Both are tools for constructing a deeper understanding of a given phenomenon. The Shared Literacies' products are independently developed graphic-symbolic representations for dance movements, which emerged through a designated methodology. These reveal new aspects of the participants' insights: in the field of movement, learners became aware of its various aspects, including the ability to analyze, think of and conceptualize the components of bodily movement; in the field of symbolic representation, participants improved their abilities to manage multifaceted information about a phenomenon, using symbolic knowledge already at hand, as well as developing new representational means. The study demonstrates the power of Shared Literacies - the integration between different artistic and general fields of thought - as a novel approach for education in the Arts.
\end{abstract}

Keywords: Art Education, Constructionism, Graphic-Symbolic Literacy, Interdisciplinarity, Movement Literacy

\section{Introduction}

The art of dance is usually perceived as a form of expression of the body moving in space and time, with or without musical accompaniment. The traditional methods of learning dance are based on either demonstration and imitation, using a set of defined concepts according to the genre studied, or independent creative processes in relation to a given topic. The graphic-symbolic component, i.e., writing and reading, which is a common teaching-learning tool in many fields of knowledge, is usually absent from dance learning.

The research presented here seeks to examine the possibility of improving learning about the body and its movement among "ordinary" students (not professional dance students) by incorporating it in alternative type of skill known to students aged 9-10 years: graphical symbolic representation of knowledge. This encounter between two fields of specializations may promote new learning channels in the field of dance and movement on the one hand, and present the contributions of learning the art of dance as part of general studies, even to those who do not intend to turn this field into their area of expertise, on the other hand. In both cases, this unique combination gives rise to an exclusive learning experience and shape knowledge of a new type. This assumption is the basis of the notion of Shared Literacies, first introduced here.

The term 'Literacy' is defined by the Oxford English Dictionary on-line first as "the ability to read and write," and second, is "the competence or knowledge in a particular area" [1]. Whereas the traditional concept of literacy has meant reading and writing alphabetic texts, more recently this term stands for elaborated knowledge and expertise or meaning-making processes in a particular field [2].

Based on this broad understanding of literacy as "a way of conveying meaning through and recovering meaning from 
the form of representation in which it appears" [3], this idea of shared literacies is emerging from the ideas of the philosopher Eliot Eisner regarding the unique opportunities embodied in art education. According to his view - the arts, as well as art education, constitute a space for conveying meaning using various modes of expression, encourage the design of personal ways for problem solving, and thus contribute to development of creativity. Such artistic practices also teach us to accept diverse solutions and interpretations with regard to a certain issue [4].

In light of these ideas, the question of the potential contribution of such encounters between artistic (movement) and cognitive (symbolic) literacies will be examined. After displaying conceptual-theoretical background, this paper will present the methodology and selected outcomes from a research study focused on such "shared literacies". In this study, elaborated knowledge from the field of the art of dance named "movement literacy", was introduced together with tasks regarding knowledge of graphic-symbolic representation, to create a novel outcome - self generated graphical-symbolic notations for dance movements. The main challenges and unique contribution of this encounter between literacies are presented and discussed.

\section{Shared Literacies: Literature Review and Theoretical Background}

The construction of shared literacies relies on several components, which will be presented in this chapter. First, the two core literacies will be introduced - movement literacy and graphic-symbolic literacy. Next, the subject of translation among representations is briefly discussed, followed by a section describing the notion of independent-generation of symbolic representations. Concluding this theoretical background is a section relating to the challenges embedded in the graphical-symbolic representations of movement.

\subsection{Movement Literacy: Conceptual and Practical Expertise in the Field of Movement and Dance}

The concept of Movement Literacy refers to a body of knowledge that integrates the practical (physical-motor) skills of movement with its theoretical understanding. In other words - the cognitive abilities which underline the knowledge of movement and its components: the body, space, and time, as well as aesthetic and emotional means of expression, and their connection to the actual movement performance [5]. The need for a definition and description of Movement Literacy as a basis for movement learning arose from years of familiarity with the accepted dance-movement teaching methods. These usually emphasize the physical-performance aspect, which is learned through either imitation, the compliance with verbal instructions, or an independent creative process, and lack the cognitive-conceptual dimension as an integral part of the learning. The integration of the practical and the theoretical aspects may provide an infrastructure for a deep understanding of the field and for various implications, such as elaborated physical performance, high-level analysis of observed movement, clever composition of dance-movement pieces, and proficient teaching methods.

The components of movement literacy - conceptualization and kinesthetic performance - are briefly presented below; the order of their presentation has no importance.

\subsubsection{Conceptualization}

At one base of movement literacy lie processes of deep knowledge with movement and its components - body, space, and time (as well as the qualitative-dynamic and aesthetic dimensions according to some of the theories), which are organized into a chosen resolution of analysis. The basis for this deep understanding relies on the conceptualization system, which according to Barsalou [6], contains people's knowledge about the world - not through general images, but through representations of the elements of experience. These may include knowledge about objects, people, situations, attributes, and relationships. These knowledge components may support a variety of cognitive operations, including naming, categorization, reasoning, argumentation, as well as the creative processes of new concepts. In turn, these basic operations lay the foundations to a variety of complex cognitive processes, such as perception, attention, memory, language, thinking, as well as socio-cultural cognition.

Altogether, with regard to the knowledge of the movement, these mental processes - generally termed "conceptualization" - greatly enhance our ability to perform actions such as, describing, discussing, documenting, composing, and instructing dance and movement, using pre-defined, unambiguous terms [7].

\subsubsection{Kinesthetic Performance (Dancing)}

The other basis of movement literacy is the practical performance of dance, meaning the physical, kinesthetic, tangible realization of knowledge, ideas and thoughts through the movements of the body in space and time. For the most part, this process requires the re-assembly of separate movement-elements into an organic whole; for example, combining arm movements with those of the legs, or combining the beginning of a dance with its continuation, in order to perform the dance piece as a whole. This proficiency poses a number of challenges for the performers, among which are a burden on memory requirements and/or physical resilience.

One major challenge that exists in all approaches to performing dance, is the recurring daily encounter with the body's constantly changing physical and mental abilities which meet the demand to bring out the shapes, feelings, and aesthetic expressions through the body; another challenge, more specific to this approach of high-level analysis, is the coordinative ability that enables the combination of the defined discrete components into a synchronized complex movement sequence [7]. This routine dance work is of a physical and artistic-expressive nature, and it is based on daily practice to maintain achieved skills and for further 
development and improvement.

The combination of the two components - the mental (conceptualization) with the practical (physical-kinesthetic performance) - produce the concept of movement literacy.

\subsubsection{Graphic-Symbolic Literacy: Generating and Decoding Symbolic Information}

Graphic-symbolic representations are a subtype of visual external representations - culturally, and mostly conventionalized - visual elements, which encode and carry meaning in various domains of knowledge. These objects, together with other kinds of external representations, hold three important features, which provide some of their basic qualities: (a) double face - they are what they are (graphic figures), and at the same time they carry meaning that is beyond their looks; (b) intentionality - they are produced by humans deliberately to carry a specific meaning; and (c) endurance - unlike the ephemerality of spoken language or disappearing movements, these entities have physical/material permanence, which allows a detachment from the process, the context and the time of production, and enables a wide range of uses at a selected time and place [8].

A review of relevant literature suggests that the concept of "symbolic literacy" is inappropriate for this paper's idea, due to its being more broadly oriented. This term usually includes references to cultural knowledge about the meaning of symbols, as an example - the dove as a symbol of peace (as is common in Western culture). Therefore, a more specific concept was chosen here, graphic-symbolic literacy, relating to proficiency in the creation and decoding of graphic-symbolic representations. This expertise is essential in today's society, as emphasized by Eilam, who wrote that "representational systems of signs and symbols help people make sense of phenomena in the world around them and reach shared meanings for these phenomena, hence contributing to the production of common cultural practices" [9]. She further claims that the growing use of visual representations in all fields of life calls for special efforts to understand their modes of creation, their meanings and how they are used to convey information [10]. The presented study, which links knowledge in the field of movement with its graphical-symbolic representations, was conducted in the framework of this idea.

\subsection{Toward Shared Literacies: Translation Between Representations and Self-Generation of Representations}

Two methods support the process of establishing a shared space between the two literacies presented - the translation among representations, which will allow the encounter and the exchange between the two, and the self-generation of graphic-symbolic representations in a domain. These are described next.

\subsubsection{Translation Between Representations}

The process of translating between different modes of representations generally means the substitution of one form of representation (tangible, conceptual or representative) with other forms. Studies that examine the possible contribution of learning experiences that involve such practices have been conducted mainly in the fields of science learning. In a publication summarizing a large number of studies, Ainsworth [11] has identified and mapped the three main functions of learning using diverse representations, and the possible rewards of translation between them. The three functions are: (a) the completion of information, (b) the constraint of possible interpretation, and (c) the construction of deep understanding. Each of these functions is based on a process of comparing the two (or more) modes of representation - in the present case, the conceptual-movement elements with the graphic-symbolic representations. The outcomes of such processes of comparison helps to identify the shared invariant features and characteristics common to both modes of representation, and to expose the differences. It invites the learner to refine and deepen their identifications in each of the domains and expand their understanding of the properties of each individual representation.

\subsubsection{Self-Generation of Representations}

The idea of students inventing their own representations has its roots in Piaget's constructivist view and in its extension, the constructionist theory of Seymour Papert [12]. This theory maintains that learning can happen most effectively when people are active in creating tangible objects in the real world. Accordingly, learners are regarded as the builders of their own cognitive tools, and knowledge is considered a personal experience to be constructed, rather than a product that may be transmitted, preserved, and re-used [13]. Thus, constructionist learning involves students drawing their own conclusions through creative experimentation and the making of social objects (such as self-generated movement notations, as is described next), which in turn will be used for discussion and partnership in the field being studied.

diSessa et al. [14] and Sherin [15] have developed this method and employed it in the domain of science and mathematics education. Alongside the application in its own right, such an idea may be used with young learners as a preliminary stage, before they are required to learn and memorize an enormous number of visual representation systems and symbols, such as graphs, tables, chemical formulas, etc. Bridging the gap between the knowledge domain and its symbolic representations establishes the learning process on existing internal resources; this process has a potential contribution to both - the specific content, as well as the general understanding of symbolic representation.

With regard to this paper - the encounter between movement knowledge (both theoretical and practical) and symbolic knowledge (which learners have accumulated throughout their lives and the intervention during the study), creates a shared space for the two literacies to interact and opens a field for novel learning opportunities.

Just before presenting some examples of the outcomes of 
such a "shared literacies" process, it is vital to understand a little more about the challenge embedded in the graphic-symbolic representation of movement.

\subsection{Challenges Embedded in Graphical-Symbolic Representations of Movement}

When attempting to represent movement using graphic-symbolic representations, one faces several challenges due to the unique characteristics of each medium: movement being three-dimensional, continuous, dynamic and ephemeral, while the symbolic representations are usually steady, discrete marks, put on a two-dimensional surface [16].

Such a challenge has been aptly described by Edward Tufte [17] who stated that "this profound and informed frustration reflects the essential dilemma of ... how to reduce the magnificent four-dimensional reality of time and three-dimensional space into little marks on paper flatlands." He continues, describing the difficulties of the process, expecting that "...perhaps one day high-resolution computer visualization... will lighten the laborious complexity of encoding - and yet still capture some worthwhile part of the subtlety of the human itinerary" (p. 119).

A potential contribution of the representation of movement through graphic-symbolic means is based on the fact that the movement is ephemeral, disappearing a moment after its execution, a fact that poses challenges of many kinds. In contrast, the stable presence of symbolic representations enables a variety of processes and the attainment of goals, such as controlled learning process, artistic reconstruction of existing dance-movement pieces for historical preservation, and novel paths towards original composition [18].

Here it is important to note briefly that throughout history a number of methods have been developed for notating movement and dance. However, unlike in music (at least in Western cultures), no system has become dominant and a major means for communication in this field.

The theoretical dance-movement knowledge used in this study was anchored in the system of the Eshkol-Wachman Movement Notation, first published in 1958 [19]. However, it is important to emphasize that during the intervention no aspects of the symbolic representation of this system was introduced to the participants.

The next section brings the research method followed by examples and outcomes from the "shared literacies" experience.

\section{Research Method: Carving the Route for Shared Literacies}

The research was carried out within the paradigm of qualitative research and, more specifically, in a dynamic process of Grounded Theory [20]. Its objectives were to improve our understanding of the process of learning movement and dance in children, focusing on the conceptual aspects of the learning process; and to better understand the available resources for the participants while developing representations of information in an unfamiliar field of knowledge.

\subsection{Participants}

Sixteen girls (ages 9-10, of average SES) volunteered to participate in an extracurricular dance class and provided their parents' consent. The dance class was conducted in a public elementary school that is located in central Israel and serves nearby rural communities.

The girls were told that the purpose of the study was to examine how children of their age visually represent movement. None of the participants had any spatial perception disability or any motoric limitations, as verified by an age-adapted ability test [21]. The 16 girls were self-organized into four groups. Each group consisted of three "developers", who generated the representations, and one "decipherer," whose role was to translate the symbolically represented movement into a kinesthetic-motor performance, with roles rotating within each group.

\subsection{Intervention}

The intervention curriculum was taught by a professional dancer, who was also a dance teacher/educator, experienced dance teacher of children, and a dance notation expert. A pre-service teacher assisted administratively, but only during some sessions.

The intervention was based on a two-part movement-content curriculum in every meeting. The first part was the introduction of the movement curriculum and its acquisition through training. All participants experienced this part at the same time. In the second part, 11 short movement sequences were presented to the "developers" only, who were then asked to write down/ draw/ notate symbols that described the movement sequence in a way that could enable the decipherer - who had not watched the demonstration - to accurately interpret and perform it. Cycles of development and decipherment of the "scripts" (the graphical text representing the movement sequence) went on until the decipherer's performance matched the original sequence. Each of the intervention lessons, spread over 29 sessions, was 60 minutes long. A detailed description of the method including the complete curriculum may be found here [22].

\subsection{Data Collection and Analysis}

Data were collected from two sources. The main source was the scripts, products of the graphic-symbolic representation process, which were filed in a group portfolio and were freely available to the participants for examination. Altogether, the four groups produced 60 scripts (initial and refined versions) for 11 movement sequences, which were analyzed to identify and describe the characteristics of the distinct symbols as well as the general layout. Another data source was the video recordings of all classes in their entirety, which captured group members' verbal and nonverbal interactions, and were transcribed and qualitatively analyzed. Analysis of the data was carried out in the process of content analysis, the main characteristics of which are the production of conclusions from the content itself on the basis of internal characteristics [23]. 


\section{Shared Literacies: Examples from the Field}

This section will present several examples from the variety of data that emerged from the study during the encounter between the movement literacy and the graphic-symbolic one These examples offer two perspectives for observation: one perspective reflects the body-movement knowledge and its translation into graphic-symbolic representation; the other focuses on the graphic-symbolic accumulated knowledge, as is expressed through the challenge of symbolically representing the moving body. All the examples are excerpts from the original full-page scripts.

\subsection{A Moving 3D Body into a Static 2D Graphic-Symbolic Representation}

As has been briefly described earlier, representation of movement by graphic-symbolic means is especially challenging due to the unique characteristics of each medium - the movement being three-dimensional, continuous, and ephemeral, while the symbolic representations are steady, discrete marks put on two-dimensional surface. The illustrations presented next exemplify representational solutions to some of these challenges, as were generated by the participants. The information in brackets indicates the group that generated each of the scripts.

\subsubsection{Two Approaches for Body Representation: Whole and Parts}

Graphic-symbolic representation of movement is usually conducted using one of two strategies: one may refer to the question - what has changed in the current whole-body position in comparison to a previous movement/position? What parts of the body have changed their position in space over the time elapsed? An alternative strategy for representation would refer only to the moving elements (body parts) in each time unit. Figure 1 presents these two strategies for representation, relating to an identical movement sequence:

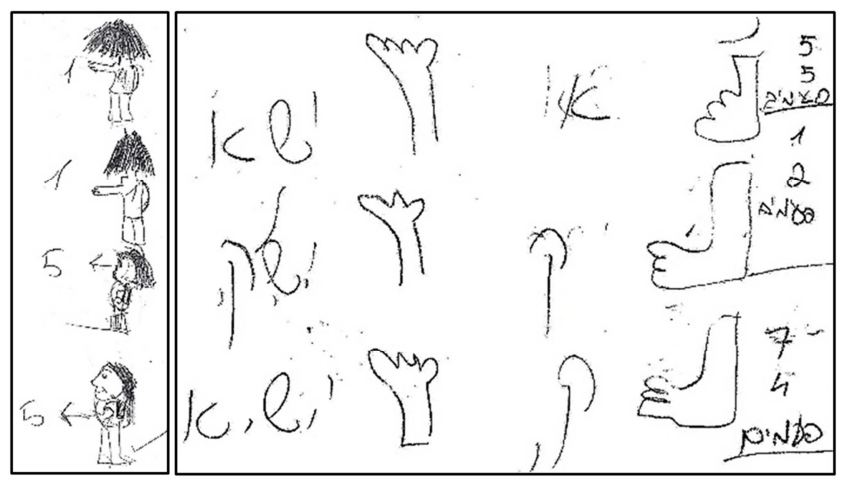

(a)

(b)

Figure 1. Representation of the same movement sequence using different representational strategies: Figure 1a, the "wholistic", presents a series of images of a whole body, in which each figure is drawn differently, presenting the changes in the relations between the limbs (Group 4); Figure 1b, the "partial", presents in a table format only the body parts that undergo a change (Group 2); the letters represent Hebrew initials for limb names.
As seen in Figure 1, the two groups chose different strategies to represent movement of the body in space and time: one (1a) depicts a series of whole-body images; each slightly different. In this method, the decipherer needs to draw conclusions vis à vis the movement action by a comparison between the images and the identification of the changes among them. On the other format (Figure 1b), more direct movement instructions are given: a "table" presents only those body parts which should take an action. Added to this are initials in Hebrew representing the names of limb and the requested movement direction (e.g., LAF = Left Arm Forward).

\subsubsection{The Challenge of Representing Movement in the Sagittal Plane (Forward and Backward)}

The movement of the body in the sagittal plane (moving forward and backward) presents a special challenge, as its representation requires a solution to the missing dimension of the writing page, the depth dimension. Figure 2 presents two possible solutions to this challenge:

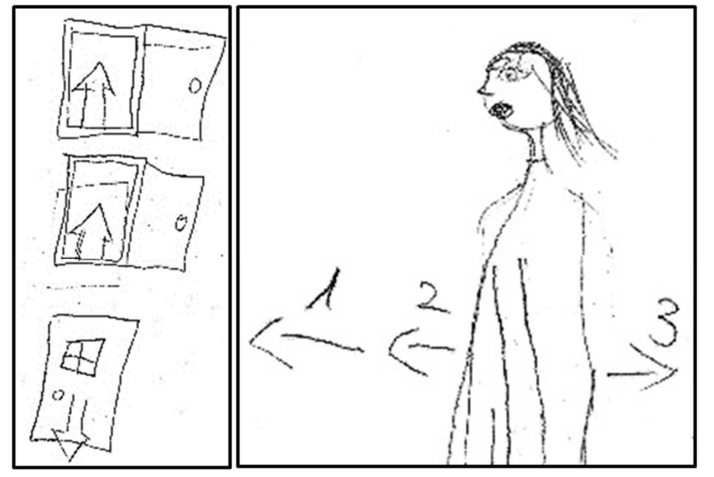

(a)

(b)

Figure 2. A sequence of three movements to directions Forward, Forward, and Backward are represented using two different modes of representations: Figure 2 a exemplifies the use of the conventional mode - arrows pointing up and down (Group 1); Figure $2 b$ presents a relational mode, where the arrow symbols are designed in relation to a central figure (Group 2).

The developers of the script presented in Figure $2 \mathrm{a}$ overcame the challenge of the depth dimension by representing the forward-back movements using a conventional means of representation - an arrow. As culturally accepted, an arrow pointing upward is usually interpreted as "forward", while an arrow pointing downward, is usually interpreted as "backward". In addition to the arrows is a unique symbolic idea: the arrows are placed in a doorframe - the "upward" arrow within an open door, as if inviting to step in (meaning "forward"), while the "downward" arrow within a frame of a closed door, as if to say "no entry" (hence, perhaps - go back). The same conventional arrow symbol is used in Figure $2 b$ in a different way: these symbols are placed in relation to a central image standing in profile (hence its forward and backward direction stays within the page) - the arrows actually designate left and right, but get their directional meanings from their relations to the central image, and the numerals represent the order of execution (Figure $2 b$ ). 


\subsubsection{Movement in the Frontal Plane (Right and Left): Symmetry of the Body and the Need for Mental Rotation}

The symmetrical structure of the body and the structural duplication of its parts involve the challenge of identifying the body sides (right-left) even when stable, prior to movement. The identification of the Right-Left directions may result from the front of the depicted figure: if the body is depicted with its back to the observer, both - the observer and the depicted figure - are facing the same direction (i.e., the sides are identical for both); if the body is depicted facing the observer, the Right-Left directions would be opposite, and one must make a "mental rotation" in order to get the directions correct.

When representing movement in the frontal dimension (to Right-Left directions) these challenges increased, as illustrated in Figure 3 below:

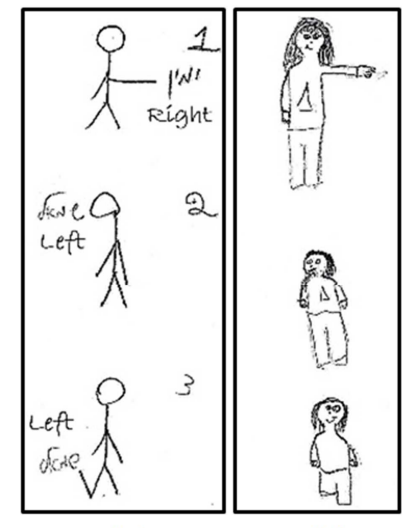

(a)

(b)

Figure 3. Representation of movement of body parts: schematically drawn figure facing away (Figure 3a, Group 1); and detailed drawn figures facing the page holder (Figure 3b, Group 4). Direction of moving parts are opposite (e.g., right arm [a] and left arm [b]).

The choice of an illustrative-iconic mode of representation by the developers of the two groups has set them up for challenges. Since the body illustrated in Figure 3a above is schematically drawn and has no clues regarding its frontal direction (either away from or towards the observer), the indication of its direction relies on the notations of direction words - Right or Left relating to the moving limbs (note: The Hebrew notations are original, those in English were added here). Based on these directions we can conclude that all images in Figure 3a are standing with their back to us, facing away. In contrast, the face and hair added to the images in Figure $3 b$ help identify their direction facing us.

And yet, despite the reversal in body-standing directions, the arm that is lifted sideways in the two upper figures is drawn in the same direction! Meaning - to the Right on 3a (according to the labels), and to the Left on $3 \mathrm{~b}$ (based on its design). This confusion is probably a mistake of the developers due to misperception and a failure in mental rotation while creating this script. With regard to the third figure in both script excerpts: two different solutions were given to represent a movement of the lower leg to the back (the missing depth dimension, as was explained in section 4.1.2): In Figure $3 \mathrm{a}$ the bent leg drawn sideways, while in Figure $3 \mathrm{~b}$ the moving part has disappeared and the (right) leg is depicted shorter than its left counterpart.

\subsection{Symbolic Knowledge Revealed and Expressed}

In this section, examples will focus on dilemmas and solutions relating mainly to the graphical-symbolic knowledge and its expression, as have been exposed during the process. These will relate to subjects regarding the accumulated culture-dependent symbolic knowledge, the double meaning of signs and the modes of their use, and the symbolic strategies for dealing with complex movement information.

\subsubsection{Directionality of Writing as Based on Accumulated Cultural Knowledge}

The directionality of writing is a subject that perhaps might be strange to those who are used to only one direction for all types of texts - verbal (written language) and symbolic (such as mathematics, musical notation, etc.). This is true for most of the Western population. In contrast, populations accustomed to additional writing directions - such as Asian languages that use vertical orientation or Semitic languages that use the right-to-left horizontal direction - understand that directionality of symbolizing and decoding text might be a significant issue.

Figure 4 and Figure 5 present some examples of how this issue of accumulated cultural symbolic knowledge was involved in the considerations determining writing directions.

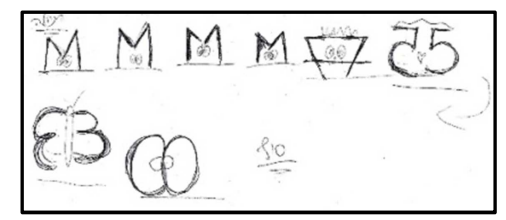

(a)

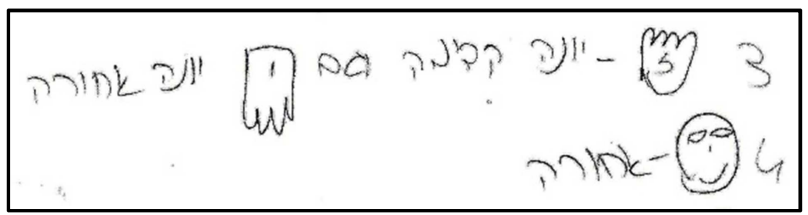

(b)

Figure 4. Different writing directions by the same group (Group 2): numeral-based text is written Left-to-Right (4a); Hebrew letter-based text is written Right-to-Left (4b).

Figure $4 \mathrm{a}$ is composed of a sequence of numerals (each is doubled as a "mirror"), running from left to right; Figure 4b is composed of a sequence of letters and words in Hebrew, running from right to left, as is customary in this language. Although not always consistent, these examples, generated by the same group of developers, allude to the existence of a relationship between the direction of writing on the script and the type of symbols used in it.

Support for this hypothesis can be found in Figure 5a-b 
below:

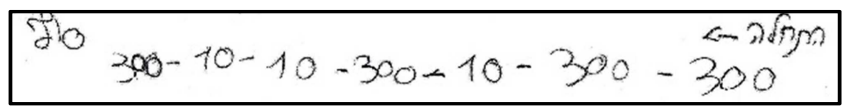

(a)

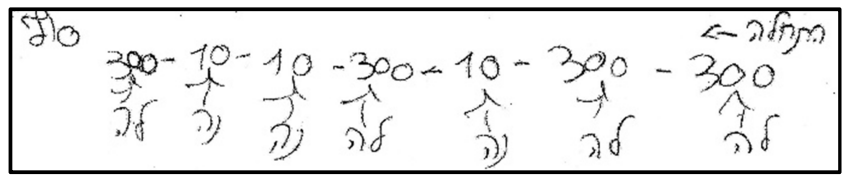

(b)

Figure 5. Initial version (5a) and its improvement (5b) present numeral-based scripts written from Right-to-Left. The improved version exposed that the developers used here the gematria method. Hence the core of the symbolic process refers to Hebrew letters, normally from Right-to-Left (both scripts by Group 2).

Figure 5a presents a script, which despite using numerals, was written from Right-to-Left. Since the decipherer failed to decode the initial version, an improvement version was crated $(5 b)$, in which it was revealed that this script was written in the gematria method, a traditional system in which numerical value is assigned to Hebrew letters. Since the core intention was to represent Hebrew letters - the first letters in the words "left" and "right" - the developers used the Right-to-Left direction.

Another example for the issue of the writing direction is shown in Figure 6:

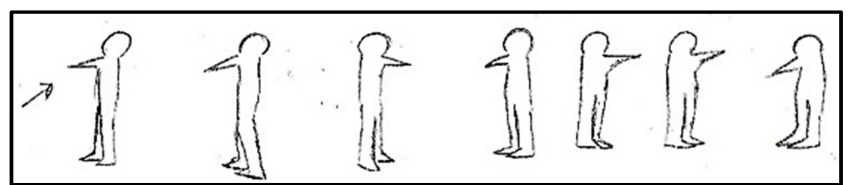

Figure 6. Representation of movement to the left and right is designed as a series of schematic figures pointing to either side. After failure in decipherment, an arrow symbol was added in the improved version to indicate the reading direction (Group 3).

Figure 6 presents a series of schematic figures that point to the sides, to represent movement to the left and to the right. The initial version (not presented here) did not include the arrow (far left), and therefore lacked an indication for the desired reading direction. As was shown earlier, Israeli pupils are acquainted with reading in both directions according to the context and the relevant language type. After an unsuccessful decipherment attempt based on reading from the right, an arrow was added by the developers to indicate reading direction (far left) and the recurring decipherment experience was successfully completed.

The use of different writing directions, as expressed in these examples, is probably the result of the accumulated knowledge of symbolic representation systems which the developers gathered throughout their experiences, within and outside the school, and the internalization of their laws. When the symbolic context lacked a clear indication, it was necessary to add a symbol indicating the reading direction.

\subsubsection{Numerals for Representing Quantity, Order and Movement Content}

Understanding the graphic-symbolic context is required not only in the context of directionality, but also when the symbols themselves could carry more than a single meaning, as is the situation with numerals.

Numerals are used in culture to represent several types of information: order, quantity, and also - unique for this study - information regarding direction in space. Examples of the different ways in which this graphic-symbolic knowledge is expressed are illustrated below.

Figure $7 \mathrm{a}$ and $\mathrm{b}$ present two script versions for the same movement sequence - movement in the forward and backward directions. Numerals here carry both quantitative and ordinal information:

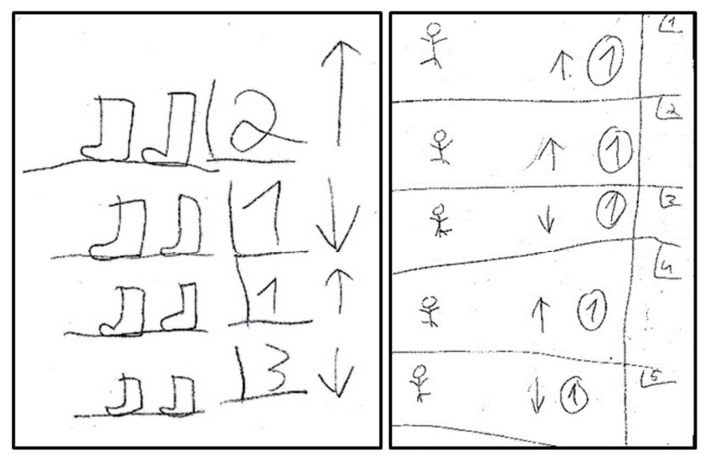

(a)

(b)

Figure 7. Numerals representing quantitative information (7a) and quantitative as well as ordinal information (7b). In $7 b$, different types of frames are designed to differentiate between the different uses of numerals: circles around quantifying numbers (in this case, always 1); a square around ordinal numbers (right corner, 1-5).

In the original version of a script representing movement sequence in forward and backward directions (7a), movements in the same direction were grouped into a single line of information (e.g., two steps forward), with the number 2 representing the number (quantity) of movements next to an arrow pointing the direction (7a, upper row). Since this idea of "formula-like" representation was not decoded, another version was created, detailing each movement unit separately $(7 b$, excerpt). In this improved version, along with the arrow pointing in the direction of movement, the number 1 was written to indicate quantity, and ordinal numbers were added at the far right of each row, representing the order in the complete sequence. The distinction between the two modes of use of the numerals is found in the addition - the quantity is circled; the order is framed by a square.

This observation suggests that the developers were aware of the multiplicity of the use of numerals for two different purposes in the same script and chose to make a clear distinction within the graphic-symbolic design.

Another example for cultural knowledge in the multiple uses of numerals is presented next. As part of the conceptual-movement training, the participants were exposed to a system for representing spatial direction as accepted in the Eshkol-Wachman Movement Notation system [19]. This 
system is somewhat similar to the compass rose with its eight directions (i.e., north, northeast, east, etc.), numbers 0-7 representing the directions. Examples of different graphic-symbolic representation of these spatial directions are given in Figure 8:

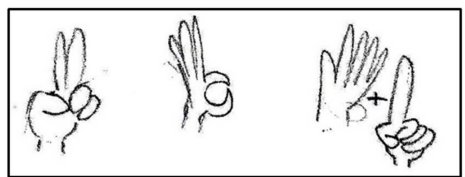

(a)

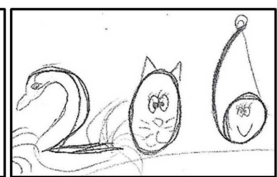

(b)

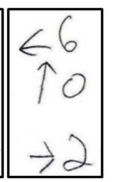

(c)
Figure 8. Representation of movement content - spatial directions (6) - (0)(2) are represented in three different ways: by digits (fingers) (8a), by decorated numerals (8b), and by arrows pointing in a similar direction of the directions represented by the numerals.

Figure 8 presents three examples for representation of the spatial directions (6), (0), (2), (which may be equivalent in certain circumstances to directions west, north, east). Each of the developers' groups chose a unique graphical mode to represent this information, and may be to differentiate this unique meaning from their "regular" numeral use (this is a hypothesis).

Figure 8a (Group 3) is based on the "digital" mode - in its original meaning, representation of numbers using fingers; in Figure $8 b$ (Group 2) each of the numerals is designed in a very ornamental way, as a character or an animal. Both these scripts' horizontal direction is Right-to-Left, which may serve as another hint that these are no "ordinary numerals".

The direction of the script in Figure 8c is vertical, a writing direction that is not under debate. Here, alongside the simple, conventional design of the numerals, arrows were added to signify the desired spatial directions. However, in the decipherment test, the added arrows confused the decoders, and it was the repeated version, without the arrows, that was easily deciphered.

Overall, the examples presented above may reflect the sensitivity and awareness of learners of approximately ten years of age of symbols and their meaning, and the need to distinguish the possible meanings through the graphic symbols.

\subsubsection{Simple Symbolic Means to Represent Movement Complexity}

In general, it can be said that the main challenge of symbolic representation is to enable expression of the complexity of the movement in its entirety in a manner that is relatively simple for depiction and decoding. One challenge of such complexity is the simultaneous occurrence of movement elements. The last example presents different approaches of representing such simultaneity. The referent movement sequence is combined of two layers of information: the movement is defined by the body's relative directions (forward and backward) which is combined with definitions of the spatial direction (0) and (2), see Figure 9:

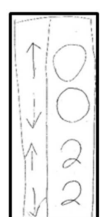

(a)

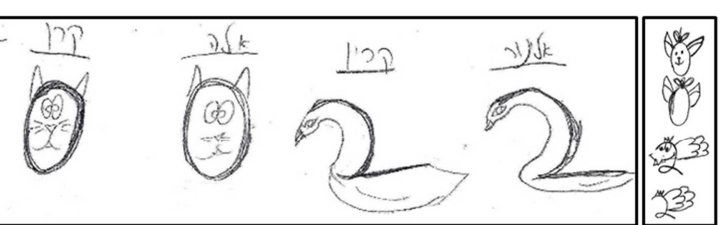

(b) (c)
Figure 9. Representation of simultaneous movement elements in three different modes. All preset combinations of the body's relative directions (forward and backward) with movement in the spatial directions (0) and (2).

The need to combine data from two separate sets of conceptual direction systems resulted in two types of graphic-symbolic solutions. A culturally widely accepted format of a table was used by two of the groups: Figure 9a (Group 1) presents a vertical table with significant columns, in one - the arrows represent the body directions, and on the other - the numerals represent the spatial directions. Reading the table should be done horizontally (in rows), thus creating a combination of the two elements into a merged movement. Figure 9b (Group 2) presents a horizonal structure of a table (although row lines are missing). The upper row contains names in Hebrew that coincide with a body direction by using the first initial of a name (an example adapted to English might be "Florence" to refer to the "Forward" direction); the lower row contains the numerals representing the spatial directions, decorated as is customary in this group. Reading of this table should be done vertically, combining information from the two rows. Both table structures are widely accepted in many domains, and it may be presumed that participants have met this format before and cleverly adapted it for the challenge of movement representation.

A unique and original approach, at least to the best of our knowledge, can be seen in the example in Figure 9c. The developers formed a set of combined symbols, each merging the two aspects into a single symbol. Thus, the digits $(0)$ and (2) of the spatial directions are designed one time with the face to the reader, meaning "forward", and another time with the back to the reader, meaning "backward". This mode of representation actually combines the specific movement-symbolic knowledge with general visual knowledge regarding the distinction between "front/face/forward" and "back/backward".

\section{Conclusion}

In light of the review of the relevant literature, the illustrations presented above display a range of products that have emerged in the new space of "shared literacies". The encounter between movement knowledge - both conceptual and practical - together with the challenges embedded in its graphic-symbolic representation, provided the learners a deeper understanding in each of the domains, with regard to its components, characteristics and possibilities: In the field of movement, learners became aware of its various components (only some of which were presented here), which included the ability to analyze, think of and conceptualize these components, and even improve their 
coordination of performance of complex movements; In the field of symbolic representation, participants improved their skills in management of complex and dynamic information of a phenomenon, using symbolic knowledge already at hand, as well as developing new representational means.

Going beyond the specific experience described, in a world characterized by the narrowing of boundaries between traditional fields of arts and knowledge, the creation of interdisciplinary fields like that presented here, and the methodology that led to it, can serve as a model for the development of innovative and relevant teaching methods.

\section{References}

[1] Literacy, N. (2019). [online] Available at: https://en.oxforddictionaries.com/definition/literacy [Feb. 28, 2019].

[2] R. Riggs Leyva. Dance Literacy in the Studio: Partnering Movement Texts and Residual Texts, Unpublished Ph.D. Dissertation, Ohio State University, 2015, pp. 16-22.

[3] E. W. Eisner. "Cognition and representation: a way to pursue the American dream?" The Phi Delta Kappan, vol. 78, no. 5, pp 348-353 [p. 353], 1997.

[4] E. W. Eisner. "The arts and the creation of mind", in What the Arts Teach and How It Shows. Yale University Press, 2002, Chapter 4, pp. 70-92.

[5] S. Ofer. (2018). "Self-Generated Notations: A Suggested Methodology of Introducing Movement Literacy," Journal of Movement Arts Literacy [On-line]. 4 (1), Article 9. Available at: https://digitalcommons.lmu.edu/jmal/vol4/iss1/9 [Feb. 28, 2019].

[6] L. W. Barsalou. "The human conceptual system" in The Cambridge Handbook of Psycholinguistics. M. J. Spivey, K. Mcrae, \& M. F. Joanisse, Eds. New York, Cambridge: Cambridge University Press, 2012, Chap. 12, pp. 239-258.

[7] S. Ofer, "Movement literacy: implementing dance notation studies into educational dance curriculum" in The Wisdom of the Many-Key Issues in Arts Education, International Yearbook for Research in Arts Education, vol. 3. S. Shonmann, Ed. Munster: Waxman Publication, 2015, pp. 117-121.

[8] L. Tolichinsky, "The multiple functions of external representations: Introduction" in Notational Knowledge: Historical and Developmental Perspectives. E. Teubal, J. Dockrell \& L. Tolichinsky Eds. Rotterdam/Taipei: Sense Publishers, 2007, pp. 1-10.

[9] B. Eilam, "Preface" in Teaching, Learning, and Visual Literacy: The Dual Role of Visual Representation. Cambridge: Cambridge University Press, 2012, p. xvii.
[10] B. Eilam, "Preface" in Teaching, Learning, and Visual Literacy: The Dual Role of Visual Representation. Cambridge: Cambridge University Press, 2012, pp. xvii-xxii.

[11] S. Ainsworth. "The educational value of multiple-representations when learning complex scientific concepts" in Visualization: Theory and Practice in Science Education. J. K. Gilbert, M. Reiner, and M. Nakhleh, Eds. New York: Springer, 2008, pp. 191-208.

[12] Y. B. Kafai. "Constructionism" in The Cambridge Handbook of the Learning Sciences. R. K. Sawyer, Ed. New York: Cambridge University Press, 2006, Chap. 3, p. 35.

[13] S. Papert and I. Har'el. "Situating constructionism" in Constructionism. S. Papert \& I. Har'el, Eds. New York: Ablex Publishing, 1991, Chap. 1.

[14] A. A. diSessa, D. Hammer, B. Sherin, and T. Kolpakowski, "Inventing graphing: meta-representational expertise in children". Journal of Mathematical Behavior, vol. 10, pp. 117$160,1991$.

[15] B. Sherin. "How students invent representations of motion", Journal of Mathematic Behavior, 19, 399-441, 2000.

[16] S. Ofer. "From movement literacy to visual literacy" in IVLA 2013 Selected Readings: Reconceptualizing Visual Literacy. N. Valanides, Ed. March, 2014, pp. 45-56.

[17] E. R. Tuft. Envisioning Information. Cheshire, CT: Graphics Press, 1990, p. 119.

[18] S. Ofer. Development of Symbolic Language to Represent Movement among Fourth Graders, Unpublished $\mathrm{PhD}$ dissertation, University of Haifa, Israel, 2009 (Hebrew with English Abstract).

[19] N. Eshkol and A. Wachman. Movement Notation. London: Weidenfeld and Nicholson, 1958.

[20] B. Glazer and A. I. Strauss. The Discovery of Grounded Theory: Strategies for Qualitative Research. Chicago: Aldine, 1967.

[21] D. Hammill, N. A. Pearson and J. K. Voress. Developmental Test of Visual Perception, $2^{\text {nd }}$ ed. Austin, Tx: Pro-ed. 1993.

[22] B. Eilam and S. Ofer. "Similar information, different representations: designing a learning environment for promoting transformational competence" in Towards a Framework for Representational Competence in Science Education, vol 11. K. L. Daniel, Ed. Models and Modeling in Science Education, pp. 31-54, Chm: Springer, 2018.

[23] E. G. Guba and Y. S. Lincoln. Effective Evaluation. San Francisco: Jessy Bass, 1981. 